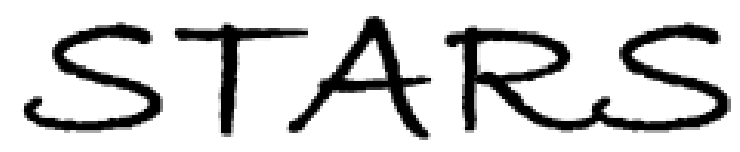

University of Central Florida

STARS

$1-1-2010$

\title{
Birefringent properties of cyclic block copolymers and low- retardation-film development
}

Weijun Zhou

Charles Diehl

Dan Murray

Kurt A. Koppi

Stephen Hahn

See next page for additional authors

Find similar works at: https://stars.library.ucf.edu/facultybib2010

University of Central Florida Libraries http://library.ucf.edu

This Article is brought to you for free and open access by the Faculty Bibliography at STARS. It has been accepted for inclusion in Faculty Bibliography 2010 s by an authorized administrator of STARS. For more information, please contactSTARS@ucf.edu.

\section{Recommended Citation}

Zhou, Weijun; Diehl, Charles; Murray, Dan; Koppi, Kurt A.; Hahn, Stephen; and Wu, Shin-Tson, "Birefringent properties of cyclic block copolymers and low-retardation-film development" (2010). Faculty Bibliography 2010s. 1021.

https://stars.library.ucf.edu/facultybib2010/1021

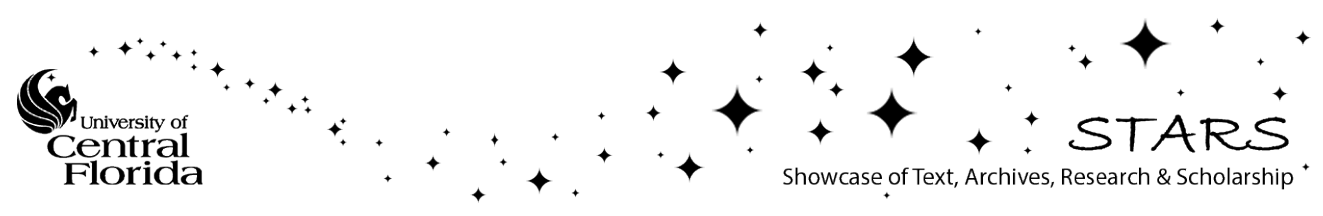




\section{Authors}

Weijun Zhou, Charles Diehl, Dan Murray, Kurt A. Koppi, Stephen Hahn, and Shin-Tson Wu 


\section{Birefringent properties of cyclic block copolymers and low-retardation-film development}

\author{
Weijun Zhou (SID Member) \\ Charles Diehl \\ Dan Murray \\ Kurt A. Koppi \\ Stephen Hahn \\ Shin-Tson Wu (SID Fellow)
}

\begin{abstract}
Cyclic block copolymers (CBCs) are a new class of optical polymers made by fully hydrogenating block copolymers of styrene and conjugated diene. This class of materials has excellent optical transparency, photostability, and good thermal resistance. By changing the copolymer composition and the resulting block-copolymer morphology, a unique set of birefringence properties can be achieved. The focus of this work was to study various sources of birefringence in block copolymers using a series of model CBC materials. One particularly interesting finding relates to the development of an ultra-low-phase-retardation CBC film. Unlike the conventional approach of using an additive or blend, a CBC film prepared by melt extrusion can readily achieve near-zero retardation in both the film plane and thickness direction. This nearly isotropic CBC film is useful as a polarizer protection film in flat-panel displays. When used as the inner protective layer of a polarizer, CBC film helps to reduce the color shift of IPS-LCDs at oblique angles and offer a wider viewing angle.
\end{abstract}

Keywords - Optical film, birefringence, low retardation, polarizer, LCD.

DOI \# 10.1889/JSID18.1.66

\section{Introduction}

Cyclic block copolymers (CBCs) are a new class of optical polymers recently developed at The Dow Chemical Company. They are made by fully hydrogenating styrene-conjugated diene block copolymers. As high-performance engineering polymers, CBC materials have many attractive property features such as excellent optical transparency, high-glass-transition temperature, good moisture-barrier property, and lightweight. ${ }^{1}$ They can be considered to belong to the same general class of polymer as cyclic olefin materials, 2,3 e.g., cyclic olefin polymers (COP) and cyclic olefin copolymers (COC), but differ from the existing cyclic olefin materials in that $\mathrm{CBC}$ can exhibit distinct block morphologies due to the microphase separation of different blocks that are covalently bonded together.

Among various unique structure-property behaviors exhibited by CBC materials, ${ }^{4,5}$ the birefringence property is particularly interesting, but not well understood. The main purpose of this paper is to investigate the birefringence property of CBC and its commercial implication for display film applications, especially for polarizer protective films.

Low retardation or zero birefringence is a critical feature needed for polarizer protection films. Triacetyl cellulose (TAC) film has been a mainstay for the polarizer industry and is used in virtually all LCDs, but has some inherent drawbacks and shortcomings. First, TAC film is very sensitive to moisture and can exhibit dimensional fluctuations in conditions where the relative humidity is changing. Secondly, conventional TAC film has a small in-plane birefringence, but has a high out-of-plane birefringence which is undesirable for high-performance LCDs. Recent efforts have been made to reduce the out-of-plane birefringence of TAC using an additive approach. ${ }^{6}$ Tagaya and co-workers have proposed a zero-birefringence polymer approach by doping negative-birefringent inorganic crystals into an acrylic copolymer. ${ }^{7}$ There are also reports of making a low-birefringent polymer composition by blending two different polymers together or by the random copolymerization of two monomers with distinctly different photoelastic properties. 8,9

Despite a significant body of research focused on the design of zero-birefringence polymer, to the best of our knowledge, very little work has been conducted to understand the birefringence property of block copolymers, particularly with respect to achieving low birefringence. In this paper, a series of CBC materials were synthesized for the purpose of understanding various sources of birefringence as well as their relative importance to the total birefringence in these microphase separated systems.

\section{Experimental}

\subsection{Materials and synthesis}

Cyclic block copolymers used in this study were synthesized at The Dow Chemical Company. A total of eight CBC materials with varying comonomer content were prepared for this study (Table 1). All but one CBC polymer (CBC-3) were made in a lab reactor. The CBC-3 polymer was produced

Expanded revised version of a paper presented at the 2009 SID International Symposium (Display Week 2009) held May 31-June 5, 2009 in San Antonio, Texas, U.S.A.

W. Zhou, C. Diehl, D. Murray, K. A. Koppi, and S. Hahn, The Dow Chemical Co., Core R\&D - New Products, 2301 N. Brazosport Blvd., B-1470, Freeport, TX 77541; telephone 979/238-1347, e-mail: Wzhou2@dow.com

S-T. Wu, College of Optics and Photonics, University of Central Florida, Orlando, FL, U.S.A.

(C) Copyright 2010 Society for Information Display 1071-0922/10/1801-0066\$1.00 
TABLE 1 - Molecular composition of CBC materials and basic property characteristics.

\begin{tabular}{ccccc}
\hline Materials & $\begin{array}{c}\text { Wt.\% of } \\
\text { styrene }\end{array}$ & $\boldsymbol{M}_{\boldsymbol{w}}(\mathbf{g} / \mathbf{m o l})$ & Polydispersity & $\boldsymbol{T}_{\boldsymbol{g}} \mathbf{( \mathbf { C } )}$ \\
\hline $\mathrm{CBC}-1$ & 55 & 77,400 & 1.08 & 109.8 \\
\hline $\mathrm{CBC}-2$ & 66 & 80,400 & 1.07 & 113.4 \\
\hline $\mathrm{CBC}-3$ & 70 & 87,200 & 1.10 & 120.7 \\
\hline $\mathrm{CBC}-4$ & 75 & 83,500 & 1.10 & 118.1 \\
\hline $\mathrm{CBC}-5$ & 80 & 94,200 & 1.11 & 121.2 \\
\hline $\mathrm{CBC}-6$ & 81 & 75,500 & 1.04 & 112.9 \\
\hline CBC-7 & 85 & 79,800 & 1.09 & 114 \\
\hline CBC-8 & 90 & 71,500 & 1.10 & 112.3 \\
\hline
\end{tabular}

with greater than 100-kg quantity to allow a large-scale film extrusion study. In all cases, the unsaturated precursor polymers were synthesized by anionic polymerization of styrene and isoprene through sequential addition of two monomers. The resulting block copolymers were fully hydrogenated using a heterogeneous porous silica supported platinum catalyst (Fig. 1). ${ }^{10-12}$ Dry polymers were obtained after a subsequent de-volatilization of the hydrogenated polymer solution. The molecular structure of each polymer was established by a combination of ${ }^{1} \mathrm{H}$ nuclear magnetic resonance (NMR) and gel permeation chromatography (GPC) measurements. Both characterizations were conducted on the polymers prior to their hydrogenation.

The molecular composition of each CBC polymer was determined by NMR spectroscopy using a Varian INOVA 300 NMR spectrometer. Polymer samples were dissolved in $\mathrm{CDCl}_{3}$ solvent at approximately $40-\mathrm{mg}$ polymer in 1-ml solvent for the analysis. A delay time of $10 \mathrm{sec}$ was used to ensure complete relaxation of protons for quantitative integrations of different chemical shifts. The percent of co-monomer in CBC (i.e., \% styrene content) and the percent of 1,4-polymerization vs. 3,4-polymerization for isoprene monomer incorporation were calculated from the NMR spectrum. The incorporation of isoprene onto the polymer backbone chain during the anionic polymerization stage was found to be predominantly through 1,4-polymerization with only about 10\% in 3,4-polymerization. The degree of hydrogenation on $\mathrm{CBC}$ polymers (i.e., post hydrogenation) was also characterized by NMR spectroscopy. Better than $99 \%$ of hydrogenation was obtained for both styrene ring and un-saturated double bonds of isoprene, suggesting the CBC polymers are fully saturated.

Molecular weights of each CBC polymer was determined from the precursor polymer prior to hydrogenation

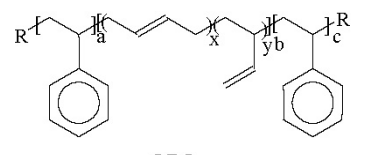

SBS

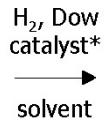

solvent

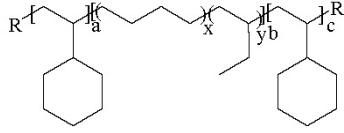

PCHE-EB-PCHE
FIGURE 1 - Schematics for cyclic-block-copolymer structure and synthesis. Hydrogenation reactions were conducted at about 600 psi of hydrogen pressure and $170^{\circ} \mathrm{C}$ using cyclohexane as the solvent. using an Agilent 1100 series GPC instrument equipped with a refractive index detector. Tetrahydrofuran (THF) was used as the solvent for dissolving the polymer and the chromatograph analysis. Calculation of the molecular weights was based on the mono-dispersed polystyrene standards. All eight CBC polymers (prior to hydrogenation) were found to be nearly monodisperse with the ratio of weight-average molecular weight $\left(M_{w}\right)$ and number-average molecular weight $\left(M_{n}\right)$ less than 1.1.

The glass-transition temperature $\left(T_{g}\right)$ for the rigid phase (i.e., hydrogenated styrene phase) of each CBC material was determined by differential scanning calorimetry (Q1000 differential scanning calorimeter, TA Instruments, Inc.). The scan rate was $10^{\circ} \mathrm{C} / \mathrm{min}$ and $T_{g}$ was determined from the second heat scan curve.

Table 1 shows the molecular composition and basic property characteristics of the eight CBC polymers used in this study. All polymers are completely amorphous with no measurable amount of crystallinity. $T_{g}$ values reported in Table 1 refer to the $\mathrm{CBC}$ rigid phase and they range from 110 to $120^{\circ} \mathrm{C}$.

\subsection{Film fabrication}

Several fabrication methods have been employed to study CBC film birefringence properties. Thin films of approximately $150 \mu \mathrm{m}$ thickness were compression molded from $\mathrm{CBC}$ materials at $250^{\circ} \mathrm{C}$. Under this molding condition, very little molecular orientation is expected in the polymer-film sample. To understand the effect of orientation on the birefringence properties of $\mathrm{CBC}$ materials, the compression molded films were uniaxially stretched by a tensile machine equipped with an environmental chamber. Micro-tensile specimens were cut out of the compression molded films for the stretching study. The stretching temperature was held constant at $135^{\circ} \mathrm{C}$ for all film samples. This is about $15-20^{\circ} \mathrm{C}$ above the glass-transition temperature of CBC materials as determined by differential scanning calorimetry. Prior to stretching, the micro-tensile bars were thermally equilibrated in the environmental chamber for 5 minutes at $135^{\circ} \mathrm{C}$. The stretching rate was set at $200 \%$ strain per sec. Once a desirable strain had been reached (e.g., $50 \%, 100 \%$, etc.), the environmental chamber was opened to allow the film sample to cool down to room temperature for removal while still under tension. The birefringence of a stretched film was measured at the same spot before and after applying the stretching.

Optical-quality CBC film was also prepared by a commercial melt extrusion process in a clean-room environment. This fabrication was only carried out on one $\mathrm{CBC}$ polymer (CBC-3) as it was available in large quantities. The melt extrusion temperature was held at $250^{\circ} \mathrm{C}$. The width of extruded film was about $1.35 \mathrm{~m}$ and the target film thickness was $60 \mu \mathrm{m}$. 


\subsection{Characterization of optical property}

Light transmission measurements were carried out on a 3mm-thick plaque prepared by injection molding. A Perkin Elmer Lambda 950 UV-Vis double-beam spectrometer was used for the study. Measurements were conducted in the wavelength range of 200-800 nm with a slit width of $2 \mathrm{~nm}$ per step.

The refractive indices of CBC polymers, $n_{0}$, were measured on a 20-mm-long $\times 8$-mm-wide CBC film specimen using a multi-wavelength Abbe refractometer (Atago DR-M2). The bottom surface of the film samples was polished so that it could make a complete contact with the main prism surface of the Abbe refractometer. A small drop of contact liquid, mono-bromonaphthalene, was used between the sample and the main prism during measurement. Care was taken to ensure the thin contact liquid was spread evenly between the sample and the main prism with no bubbles.

Optical retardation and birefringence of film samples were measured on an EXICOR ${ }^{\mathrm{TM}}$ 150ATS (Hinds Instrument, OR) birefringence measurement system at three wavelengths: 436, 546, and $633 \mathrm{~nm}$. The in-plane retardation $\left(R_{0}\right)$ and thickness direction retardation $\left(R_{t h}\right)$ are defined as follows:

$$
\begin{gathered}
R_{0}=\left(n_{x}-n_{y}\right) \times d, \\
R_{t h}=\left(\frac{n_{x}+n_{y}}{2}-n_{z}\right) \times d,
\end{gathered}
$$

where $d$ is the film thickness and $n_{x}, n_{y}$, and $n_{z}$ represent the refractive index along three principal axes $x, y$, and $z$, respectively. The directions $x$ and $y$ define two mutually orthogonal axes in the film plane, and $z$ is along the film thickness direction. The direction along which the refractive index is higher in the film plane is denoted as the slow axis. In this paper, the $x$-direction is assigned to be the slow axis.

$R_{0}$ was measured with incident light normal to the film surface. Determination of the $R_{t h}$ values was based upon retardation measurements made with incident light normal to the film surface (i.e., $R_{0}$ ) and at an oblique incidence angle of $40^{\circ}\left(R_{40}\right)$. Measurement of $R_{40}$ was made by tilting the film around the slow axis at $40^{\circ}$. By solving the following four equations, $n_{x}, n_{y}$, and $n_{z}$ can be determined and substituted into Eq. (2) for calculating $R_{t h}$ values

$$
\begin{gathered}
n_{x}+n_{y}+n_{z}=3 n_{0}, \\
\left(n_{x}-n_{y}\right) \times d=R_{0}, \\
\left(n_{x}-\frac{n_{y} n_{z}}{\sqrt{n_{z}^{2} \cos ^{2} \theta+n_{y}^{2} \sin ^{2} \theta}}\right) \times \frac{d}{\cos \theta}=R_{40}, \\
\sin \theta=\frac{\sin 40^{\circ}}{n_{0}},
\end{gathered}
$$

where $n_{0}$ is the nominal refractive index of the polymer and $\theta$ is the direction of light propagation within the film when it is tilted at $40^{\circ}$.

\subsection{Surface characterization}

The surface quality of CBC films prepared by melt extrusion was examined by atomic force microscopy (AFM). Film surface topography was captured at ambient temperature using a Digital Instruments Multi-Mode AFM equipped with a NanoScope IV controller. Nano-sensor probes with a spring constant of $55 \mathrm{~N} / \mathrm{m}$ and a resonant frequency in the vicinity of $167 \mathrm{kHz}$ were used for phase imaging. The samples were imaged at a frequency of $0.5-2 \mathrm{~Hz}$ and a set point ratio of $\sim 0.8$. Film-surface roughness (i.e., z-direction height) was calculated on the sample size of $40 \times 40 \mu^{2}$. The geometric mean deviation of all points on the surface from the mean, commonly denoted as root-mean-square (RMS) roughness, is reported in this study.

\section{Results and discussion}

\subsection{General optical properties of CBC polymer}

CBC polymers are fully saturated olefin block copolymers with excellent light transmission. The overall transmission characteristics are similar to those of cyclic olefin polymers (COP). ${ }^{2}$ As shown in Fig. 2, a 3-mm-thick injection molded CBC plaque shows better than $92 \%$ light transmittance across the entire visible wavelength range from 380 to 780 $\mathrm{nm}$. This level of optical transmittance is a few percent higher than those of optical polymers such as polycarbonate (PC) and polyethylene terephthalate (PET) and is close to being best in class among all optical polymers.

CBC polymers also have very low haze. For an opticalquality CBC film of $80 \mu \mathrm{m}$ thickness or thinner, haze was measured to be lower than 0.2. In comparison, the haze of TAC and PC optical film is at about 0.3 , whereas the haze of

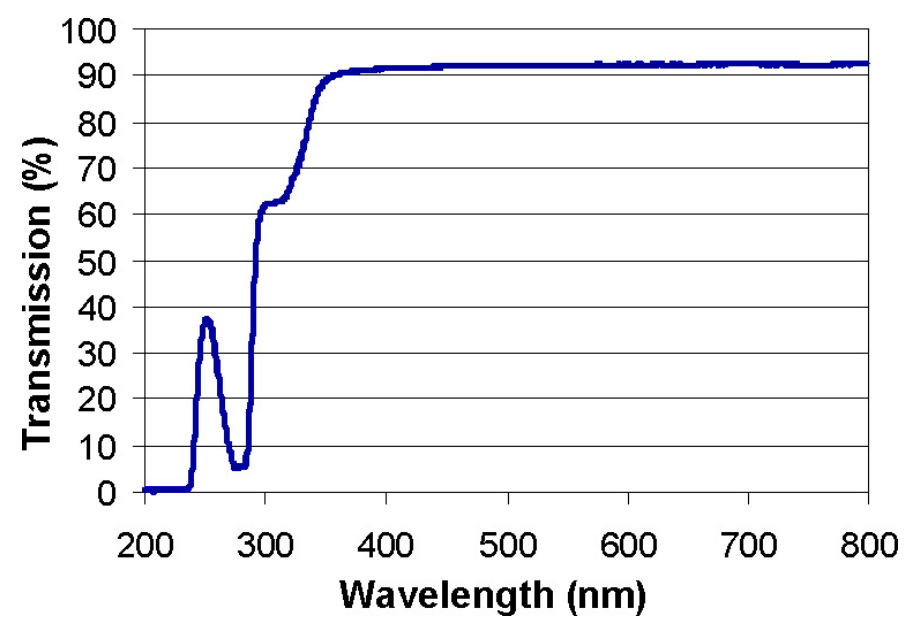

FIGURE 2 - Light-transmission spectrum of a 3-mm-thick injection molded CBC plaque. 
PET film is considerably higher and reaches nearly 1.8 at about the same film thickness. ${ }^{13}$

The color of CBC polymers and fabricated articles is also superior. No inherent color bodies exist in the CBC molecular structure that could otherwise lead to yellowing or other light stability problems commonly encountered during the long term use of aromatic polymers (e.g., PC). The yellowness index (YI) of a CBC film at $80 \mu \mathrm{m}$ thickness or thinner is typically smaller than 0.3 . In contract, a conventional TAC film has a YI value about 1.0 and is visibly more yellow than a CBC film. These excellent optics properties demonstrate a good suitability of CBC polymers for use in display film applications.

The refractive index of the CBC-3 polymer was measured to be approximately 1.507 at room temperature and $589 \mathrm{~nm}$ (D-line). This is slightly higher than both PMMA and TAC materials that have a refractive index of about 1.49. Variation of the comonomer ratio (i.e., styrene vs. isoprene) does not significantly affect the refractive index value of CBCs. This is due to the close match of the refractive indices between the hydrogenated styrene phase $(n=1.51)$ and the hydrogenated isoprene phase $(n$ $=1.486$ ).

Figure 3 shows the effect of wavelength on the refractive index properties of CBC-3 polymer. The solid curve represents the fit of refractive index data to the two-coefficient Cauchy equation: ${ }^{14}$

$$
n(\lambda)=A+\frac{B}{\lambda^{2}} .
$$

There is a reasonably good fit between the experimentally measured refractive-index values and the Cauchy equation. For the particular polymer shown in Fig. 3, values of $A$ and $B$ were determined to be 1.4915 and 0.0042 $\left(\mu \mathrm{m}^{2}\right)$, respectively. This set of dispersion values will be used for the color-shift calculation of CBC polarizer film in Sec. 3.5.

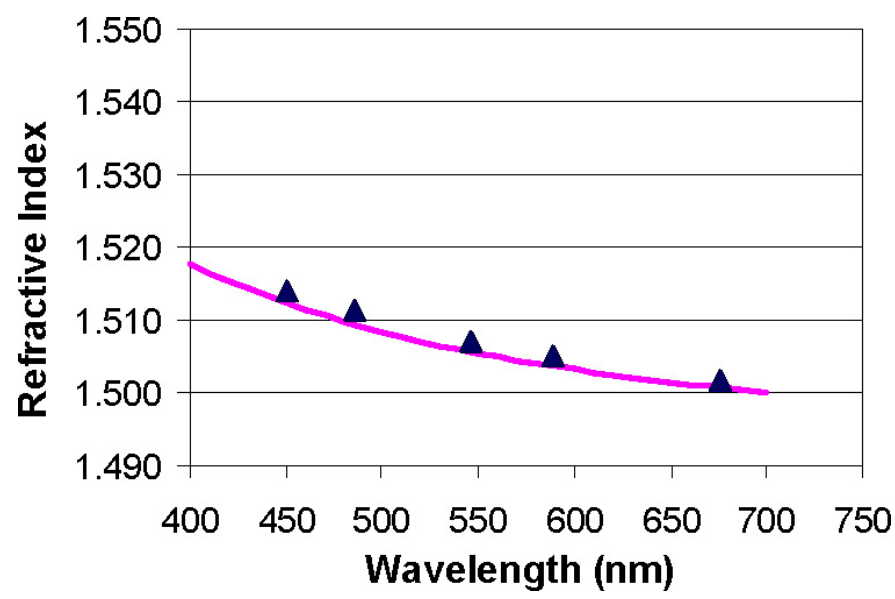

FIGURE 3 - Refractive index of CBC-3 polymer as a function of wavelength.

\subsection{Birefringence properties of CBCs}

The birefringence properties of a polymer can be quite complex. Both molecular structure and processing conditions affect the measured birefringence in a fabricated polymer film or article. Figure 4 shows the birefringence of CBC films prepared by compression molding as a function of rigid co-monomer content (i.e., styrene). In all cases, the amount of birefringence is very low $\left(\mathrm{ca} .10^{-6}\right)$. A slight decrease in birefringence is observed with an increase of styrene content. A low birefringence is expected for thin films of CBC materials made by compression molding because such a fabrication process should not lead to any appreciable amount of molecular orientation.

Two possible root causes may explain the effect of co-monomer content on birefringence shown in Fig. 4: (1) block-copolymer microphase separated structure and (2) individual chain polarizability. Polymer films prepared by compression molding are often not completely isotropic and there tends to be a preferential (albeit minor) alignment of block-copolymer domains. Such alignment is more likely in materials with a high degree of microphase separation. CBC materials with relatively low styrene content (e.g., CBC-1 and $\mathrm{CBC}-2$ ) are more prone to microphase separation because of their more symmetrical blocky structure. Microphase separated chains are not in random coil configurations, and if these extended chains are arranged in a macroscopically anisotropic matrix (i.e., preferential domain alignment), the resulting material can exhibit birefringence even though the birefringence of an individual chain is low (or nearly zero).

The second possibility is that $\mathrm{CBC}$ polymers with relatively high rigid co-monomer content are slightly more isotropic. In another words, the difference between the polarizability along the chain axis $\left(b_{1}\right)$ and perpendicular to the chain axis $\left(b_{2}\right), \Delta b=b_{1}-b_{2}$, becomes smaller when increasing the rigid comonomer content in CBC materials.

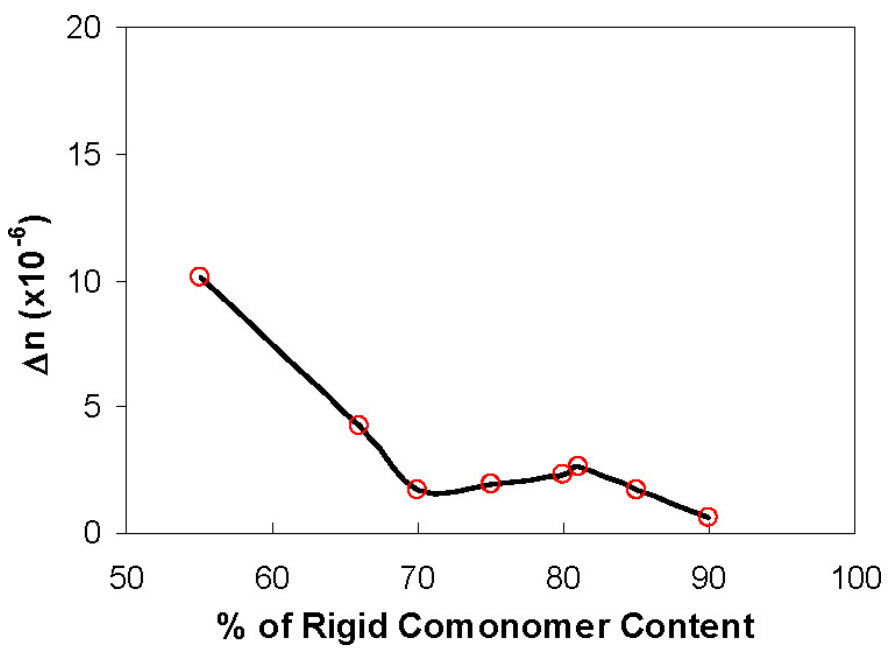

FIGURE 4 - Birefringence of CBC polymers at pseudo isotropic state as a function of styrene monomer content. The measurements were conducted on thin films of approximately $150 \mu \mathrm{m}$ thickness prepared by compression molding. 
According to a statistical segmental model analysis, ${ }^{15,16} \Delta b$ for the chain segment consisting of hydrogenated styrene units is calculated to be negative at $-2.1 \times 10^{-12}$, whereas for the chain segment consisting of hydrogenated isoprene units, $\Delta b$ is positive at $19.6 \times 10^{-12}$. It is noted here that the negative $\Delta b$ for the rigid block is primarily due to the pendant cyclohexane ring structure. If ignoring the volumetric difference between two monomer units, a simple mixingrule analysis suggests that $\Delta b$ approaches zero at about $90 \mathrm{wt} . \%$ of rigid comonomer content, representing the most isotropic state a CBC chain can possibly have. When the rigid monomer content is less than $90 \mathrm{wt}$ \%, a CBC polymer would be expected to display a positive birefringence. On the other hand, if the rigid monomer content is greater than $90 \mathrm{wt} . \%$, there is a tendency for CBC polymer to exhibit negative birefringent behavior. Indeed, by varying the co-monomer content, CBC polymer exhibits a crossover from positively birefringent to negatively birefringent behavior. However, a CBC polymer with greater than 90 wt.\% of rigid co-monomer content tends to be very brittle and is not suitable for use in display film applications.

The influence of fabrication and, in particular, the orientation effects on the birefringence behavior of CBC materials were also investigated. This knowledge is important for designing a block copolymer that is suitable for manufacturing an optical film by melt extrusion, a process that would inevitably lead to some degree of molecular orientation and possibly block-copolymer morphological alignment in the film. Various films that were uniaxially stretched at an elevated temperature by a tensile-stretching machine were used for this study. Prior to stretching, films were prepared by compression molding with a target thickness of $150 \mu \mathrm{m}$ and then cut into micro-tensile bars. The stretching temperature was kept constant at $135^{\circ} \mathrm{C}$ for all the $\mathrm{CBC}$ films. The elongation relative to the original film length (i.e., stretching ratio) varied from $30 \%$ to $200 \%$. After the stretching operation, the film specimen was taken out of the tensile stretching machine and left at the room temperature for 48 hours before optical measurements were performed. The birefringence at the middle point of the specimen was measured as a function of the stretching ratio.

The stretched CBC films were found to have a birefringence on the order of $10^{-4}$. This is about two orders of magnitude higher than the unstretched state, indicating a strong effect of molecular orientation on the measured optical anisotropy. When the stretching ratio is at about $130 \%$, the increase in birefringence begins to level off. Further stretching of the film at higher stretching ratio either does not result in any substantial increase in the measured birefringence or the film-stretching operation becomes too unstable to allow for reproducible measurements. For this reason, the birefringence at the stretching ratio of $150 \%$ will be compared for CBCs with different co-monomer content.

Figure 5 shows the birefringence as a function of rigid co-monomer content for various stretched CBC films. A rather interesting and complex relationship is observed.

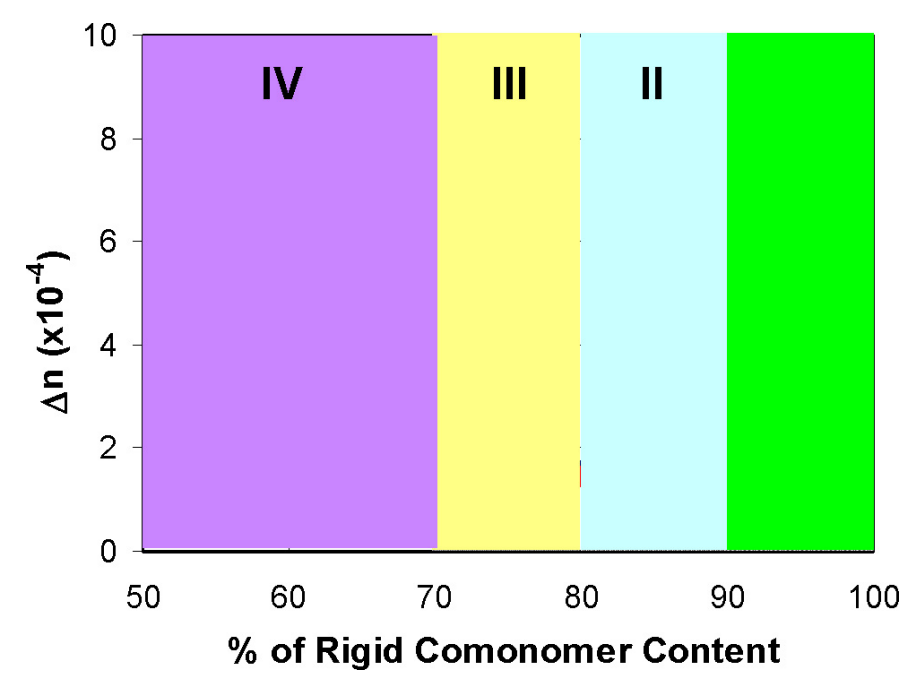

FIGURE 5 - Birefringence of $\mathrm{CBC}$ polymers at oriented state as a function of styrene monomer content. The measurements were conducted on thin films that were subjected to a uniaxially stretching orientation at the stretching temperature of $135^{\circ} \mathrm{C}$. $\mathrm{CBC}$ tends to form distinctly different morphology at four shaded regions.

Most evidently, a local maximum occurs for the CBC-4 polymer having $75 \mathrm{wt}$. \% of rigid comonomer content. If this particular data point is neglected, there is an overall trend of the birefringence decreasing with the increase of rigid comonomer content in the compositional space investigated. This is similar to what is observed on un-stretched films due to the polarizability difference (Fig. 4). Thus, a different mechanism would have to be involved to explain the considerably higher birefringence measured on the stretched CBC-4 film.

One possible cause is the contribution of form birefringence $\left(\Delta n_{f}\right)$ for the total birefringence of CBC polymers. Form birefringence arises as a result of multiple phases in the material in which each phase has a different refractive index. Additionally, at least one phase needs to be geometrically anisotropic (e.g., cylinder or plate). ${ }^{17,18}$ Thus, a homopolymer or random copolymer would not exhibit any form birefringence, but a block copolymer like CBC can exhibit distinct microphase separation between two blocks that leads to a considerable amount of form birefringence. Of the various $\mathrm{CBC}$ polymers studied here, it is believed that CBC-4 is the only CBC polymer included in this investigation that tends to form a cylindrical morphology with the minor phase (i.e., hydrogenated isoprene blocks) forming dispersed cylinders in the continuous matrix consisting of hydrogenated styrene blocks. ${ }^{19}$ The cylinder morphology is known to produce form birefringence, and its effect will be enhanced when cylinders are uniaxially oriented (e.g., by a stretching orientation process). On the contrary, CBC materials of other composition shown in Table 1 are less prone to exhibit form birefringence effect because of their non-cylindrical morphology.

The magnitude of form birefringence for CBC-4 polymer can be calculated by the following equation: 


$$
\Delta n_{f}=n_{\|}-n_{\perp}=\frac{\mathrm{v}_{1} \mathrm{v}_{2}\left(n_{1}^{2}-n_{2}^{2}\right)}{2 n_{\|}\left[\left(\mathrm{v}_{1}+1\right) n_{2}^{2}+\mathrm{v}_{2} n_{1}^{2}\right]},
$$

where $n_{\|}$and $n_{\perp}$ are the apparent refractive index of polymers in the direction parallel and perpendicular to the cylinder axes, respectively; $v_{1}$ and $n_{1}$ are the volume fraction and refractive index of the rigid (matrix) phase; and $v_{2}$ and $n_{2}$ are the volume fraction and refractive index of the soft (cylinder) phase. The form birefringence is calculated to be $\Delta n_{f} \sim 1.1 \times 10^{-4}$. This value is apparently non-negligible, but still relatively small compared to the total birefringence of CBC- 4 at $6.7 \times 10^{-4}$. Thus, the form birefringence alone can not account for the considerably higher birefringence of CBC-4 relative to other compositions (Fig. 5).

It is now clear that the molecular orientation is probably still the dominant mechanism for the birefringence in all CBC polymers. Furthermore, since the intrinsic birefringence (referring to the maximum birefringence of a polymer chain under perfectly oriented condition) should follow a linear function of comonomer content, the non-monotonic behavior of the measured birefringence shown in Fig. 5 suggests the stretched films possess different degrees of orientation after the stretching. This is likely when considering the less than desirable stretching protocol used in this study. Equally important, the variable rate of polymer-chain relaxation during the stretching at a fixed stretching temperature of $135^{\circ} \mathrm{C}$ can also affect the degree of orientation that can be retained in the solid film sample. It is also conceivable that the competition between stretching orientation and thermodynamically driven microphase separation may have resulted in a different degree of optical anisotropy for CBC materials possessing different morphology.

One interesting finding worthy of note is the birefringence of CBC-3 material. This material exhibits a lower birefringence than both CBC-2 and CBC-4, but it has an intermediate comonomer content. We suspect CBC-3 might be inherently more isotropic due to the possible formation of a bi-continuous gyroid phase. Further evidence supporting such a morphology is the fact that CBC-3 exhibits an excellent balance of thermal and mechanical properties. However, there is no direct evidence (e.g., microscopy or scattering) confirming the presence of gyroid morphology in CBC-3.

\subsection{Melt extrusion behavior of $\mathrm{CBC}$ polymer}

The melt extrusion process for making thin films with properties and quality appropriate for use in polarizer applications has presented a series of challenge that was not solved until very recently. A viable melt process that prepared usable polarizer film was first demonstrated on COP by Zeon Corp. ${ }^{13}$ Similar to COP, we found CBC materials also exhibit very good film forming ability across a wide compositional range. Films with thickness ranging from 20-100 $\mu \mathrm{m}$ or higher can be readily cast from a CBC polymer using a melt-extrusion process.

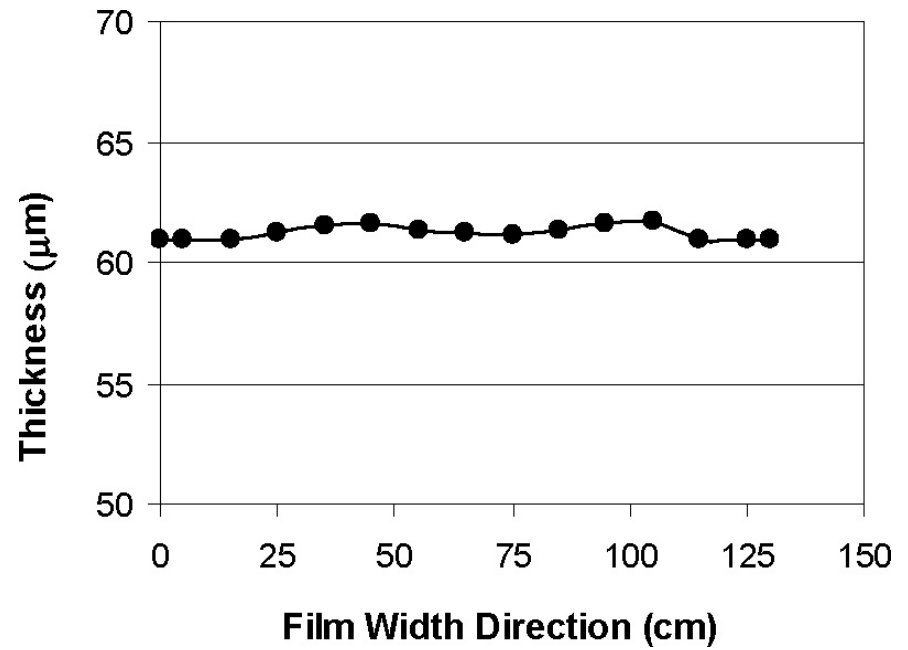

FIGURE 6 - Thickness profile of a CBC-3 film prepared by a commercial melt-extrusion process.

Figure 6 shows the film-thickness profile of a 1340nm-wide CBC-3 film. Thickness measurements were made along the film width direction. The average film thickness was measured to be $61.2 \mu \mathrm{m}$ with a standard deviation of about $0.26 \mu \mathrm{m}$. No visible die lines or streak-like cosmetic defects along the machine direction and the transverse direction were observed on the film, suggesting the melt-extrusion cast process is inherently capable of manufacturing thin film (i.e., less than $100 \mu \mathrm{m}$ ) with a high degree of uniformity for display applications.

CBC films prepared by melt casting also exhibit excellent surface uniformity at the microscopic level. Figure 7 shows the surface morphology of the CBC-3 film measured by atomic force microscopy (AFM). The film side directly contacting the cast roller [Fig. 7(a)] was found to be very smooth. The root-mean-square average roughness $\left(R_{q}\right)$ was calculated to be only about $1.7 \mathrm{~nm}$. The other side of the CBC film was shown to be slightly rougher [Fig. 7(b)], nevertheless, the $R_{q}$ value is still relatively low at $5.8 \mathrm{~nm}$. This level of surface roughness is better than a standard TAC film made by the solution casting process. $R_{q}$ values measured on the two sides of a TAC film (e.g., Fujifilm TD80ULM) are at 6.2 and $6.7 \mathrm{~nm}$ using a similar AFM surface morphology analysis.

In many respects, a melt extrusion process is advantageous over the conventional solution casting process for making a polarizer film. First and foremost, the melt-extrusion process reduces the total cost of manufacturing an optical film and it is more environmental friendly. Current TAC film manufacturing involves many steps and is inherently complex and expensive, partly due to the need to comply with environmental regulations of solvent emission and handling. Secondly, TAC film with thickness less than $40 \mu \mathrm{m}$ is very difficult to make. As a result, TAC film is becoming a less-desirable choice for use in portable devices such as cell phones, PDAs, etc., where a slim and light display design is necessary. The excellent film-forming ability of CBC polymer can potentially be used in making thinner films that 

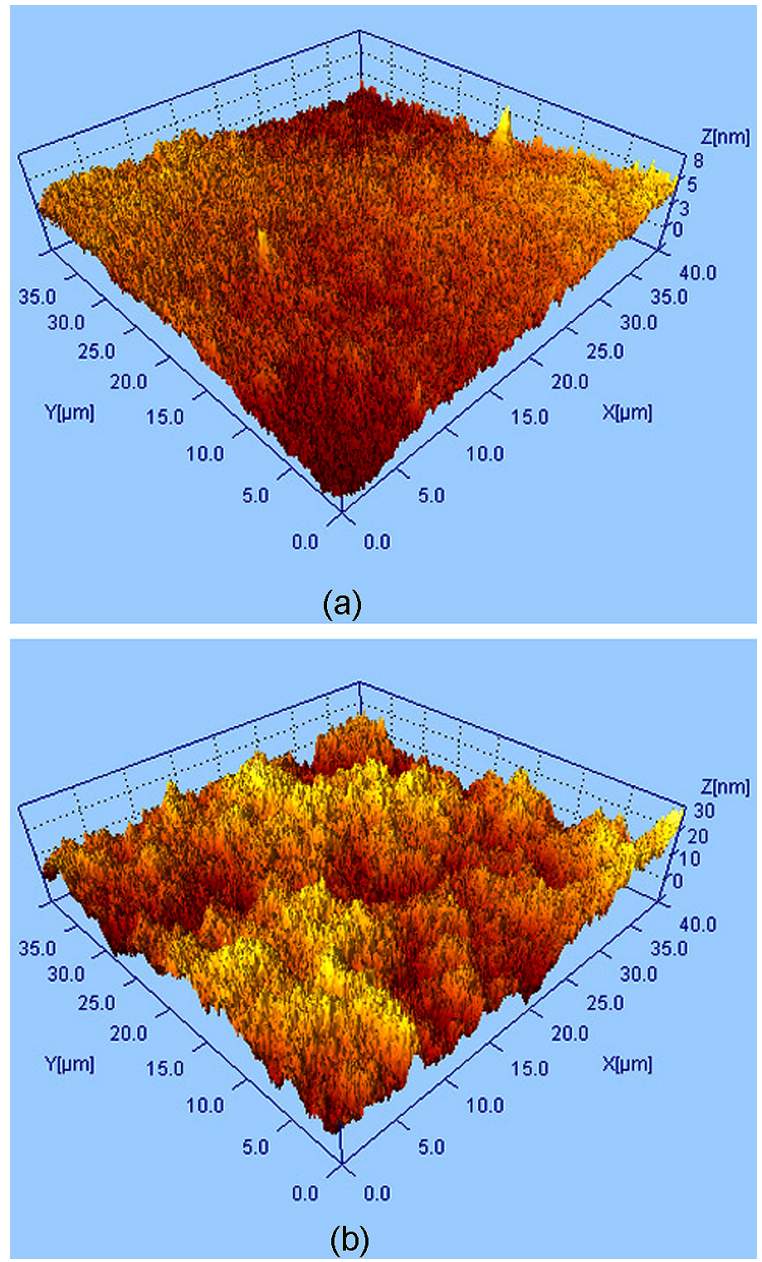

FIGURE 7 - AFM surface morphology of CBC-3 film: (a) film side contacting the extrusion casting roller; (b) opposite side of the film.

have not been possible by TAC. Thirdly, the possibility of micro-patterning an optical film during the melt-extrusion process, an option not feasible in solution cast, opens up an additional opportunity for cost reduction and size compaction of the LCD.

\subsection{Results of low-retardation CBC film}

Low-retardation CBC film with high optical quality was successfully made by melt casting CBC-3 material. As we discussed in the earlier sections, CBC- 3 provides a good balance in terms of melt-extrusion processing, physical properties, and low birefringence. Thus, the remaining sections will mainly focus on films made from the CBC-3 material.

Figure 8 shows a comparison of film retardation at different light incidence angles for CBC-3 film and a conventional TAC film. The retardation was measured at a single wavelength of $633 \mathrm{~nm}$. Unlike the TAC film which exhibits significant dependence on light incidence angle, the optical retardation of CBC-3 film is essentially constant at all incidence angles, suggesting the CBC film is optically isotropic. Moreover, the optical retardation of CBC-3 film is very low

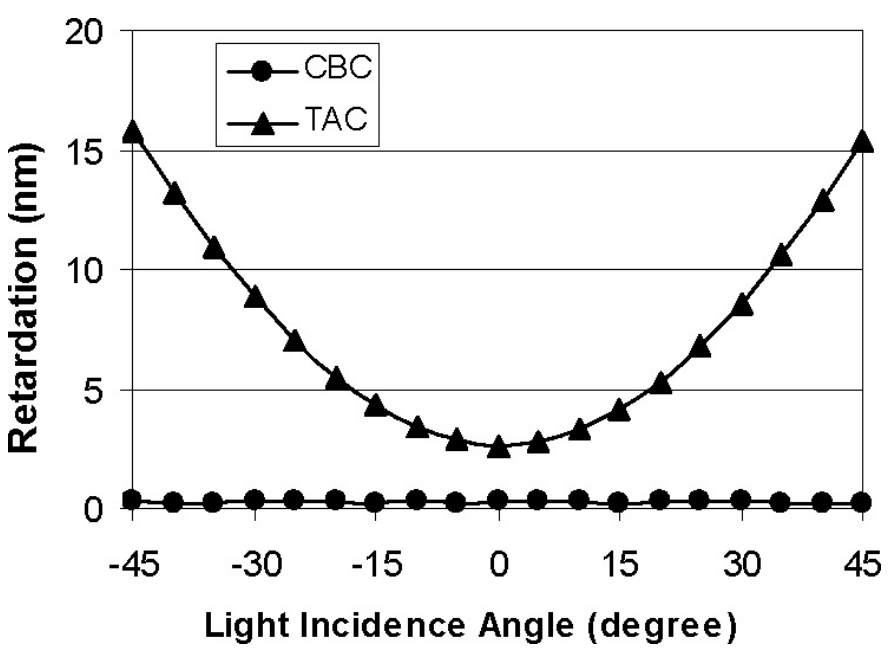

FIGURE 8 - Optical retardation of CBC-3 film and a conventional TAC film measured at different light incidence angles and the wavelength of $\lambda=633 \mathrm{~nm}$.

and less than $0.3 \mathrm{~nm}$ at all incidence angles. Thus, it should be very suitable for polarizer-film use.

CBC-3 film also exhibits a higher degree of retardation uniformity across a large film area. As shown in Fig. 9, both in-plane retardation $\left(R_{0}\right)$ and thickness direction retardation $\left(R_{t h}\right)$ were found to be nearly zero across the entire film width direction. The average $R_{0}$ is about $0.2 \mathrm{~nm}$ with a standard deviation of less than $0.1 \mathrm{~nm}$. The average $R_{t h}$ is approximately $0.1 \mathrm{~nm}$ with a standard deviation of less than $0.1 \mathrm{~nm}$. To the best of our knowledge, this level of optical retardation is the lowest among all display films. It is also worth noting here that both $R_{0}$ and $R_{t h}$ are stable after exposure of CBC-3 films to moisture because of the excellent low moisture absorption properties of CBC materials.

The wavelength dependence of $R_{0}$ and $R_{t h}$ for CBC-3 film and a conventional TAC film are compared in Fig. 10. Neither retardation property shows any measurable wavelength dispersion effect for CBC-3 film; $R_{0}$ and $R_{t h}$ are essentially unaffected by the change in wavelength. On the contrary, both $R_{0}$ and $R_{t h}$ of TAC film exhibit strong wavelength

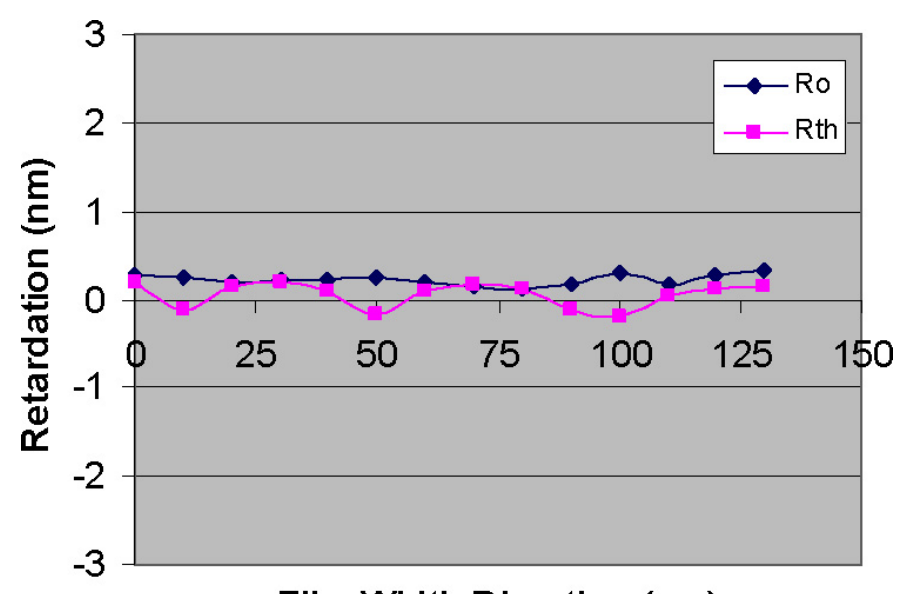

Film Width Direction $(\mathrm{cm})$

FIGURE 9 - Optical retardation of CBC-3 film across the entire film width direction. 

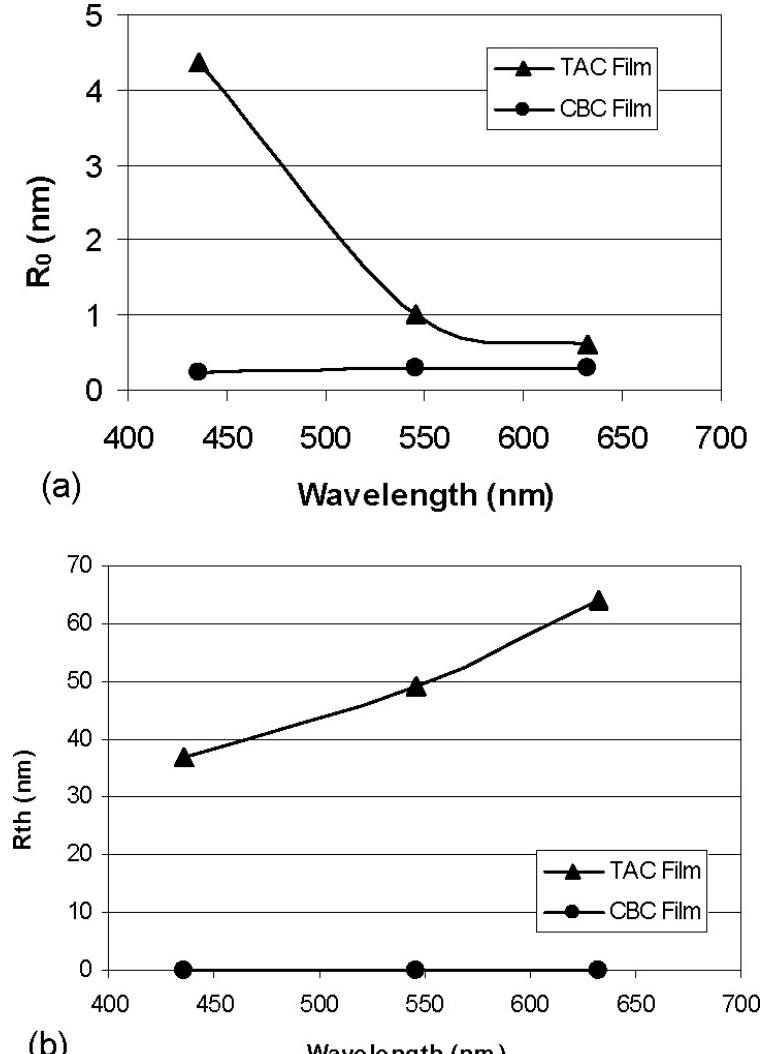

FIGURE 10 - In plane retardation $R_{0}$ and thickness direction retardation $R_{\text {th }}$ for CBC-3 film and a conventional TAC film at three different wavelengths: (a) $R_{0}$ as a function of wavelength; (b) $R_{\text {th }}$ as a function of wavelength. The measurements were made on a CBC-3 film of $60 \mu \mathrm{m}$ thickness and a TAC film of $80 \mu \mathrm{m}$ thickness.

dependence; $R_{0}$ decreases with the increase of wavelength, whereas $R_{t h}$ increases with the increase of wavelength. In general, the strong wavelength dependence shown by TAC film is undesirable for wide-view performance and has to be addressed during LCD system design.

Additionally, the $R_{t h}$ value of TAC film is rather high and reaches about $50 \mathrm{~nm}$ at $\lambda=550 \mathrm{~nm}$. This is suspected to be due to the out-of-plane orientation of the cellulose ring structure of the TAC molecule. The high $R_{t h}$ values of the TAC film, when used as a polarizer protective film, is known to cause a substantial color shift in IPS-LCD. ${ }^{6}$ In contrast, the $R_{t h}$ of the CBC-3 film is roughly $0.1 \mathrm{~nm}$. The extremely low $R_{t h}$ value of CBC-3 film should be advantageous for use as a polarizer protection film in general and particularly in an IPS-LCD for improved wide view performance.

\subsection{LCD simulation results}

The large color shift caused by the non-negligible retardation of TAC protective film in a polarizer assembly has been a well-known problem in IPS-LCD cells. To understand the potential benefit of using a completely isotropic film like CBC-3 for polarizer applications, the image-viewing characteristics were studied through an optical simulation using a

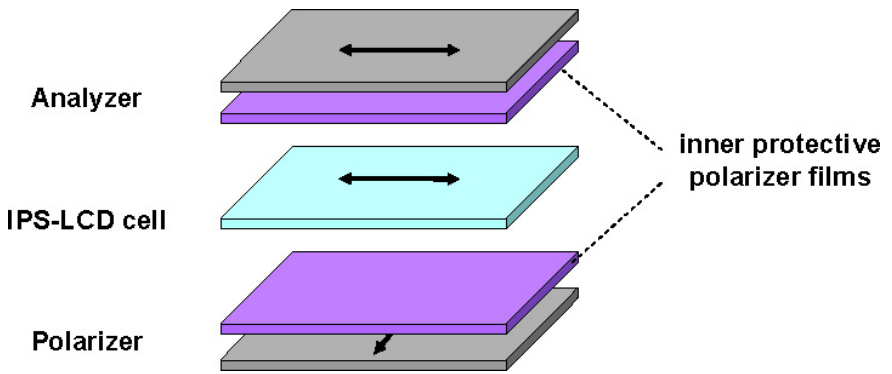

FIGURE 11 - Schematic layout of the IPS-LCD cell for the optical simulation.

standard IPS-LCD structure described by Lu et al. ${ }^{20}$ Figure 11 is the simple layout of the IPS-LCD cell used for our simulation. The active component of the cell used for these simulations have the following characteristics. The liquidcrystal mixture MLC-6692 from Merck is homogeneously aligned in the LCD cell. The pre-tilt angle of LC director is $\sim 2^{\circ}$ and the rubbing angle is at $10^{\circ}$ with respect to the longitudinal direction of the electrodes. The electrode width, the electrode gap, and the liquid-crystal cell gap are maintained at 4,8 , and $4 \mu \mathrm{m}$, respectively.

Figure 12 shows the calculated CIE 1931 chromaticity diagram of an IPS-LCD cell using CBC-3 film $\left(R_{0}=0.2 \mathrm{~nm}\right.$ and $R_{t h}=0.1 \mathrm{~nm}$ ) as the inner protective film for both the bottom polarizer and the top analyzer. A standard cold-cathode fluorescent-lamp (CCFL) source from 380 to $780 \mathrm{~nm}$ was used for the calculation. The incident light was at a $40^{\circ}$ angle with respect to the cell normal direction and scanned across the entire azimuthal range. Results show a relatively minor color shift. The color shift $\left(\Delta u^{\prime} v^{\prime}\right)$ was calculated to be about 0.128 across the entire viewing angles.

A similar color-shift calculation was conducted when using two conventional TAC films as the inner protective films for both polarizer and analyzer. Results for a TAC film

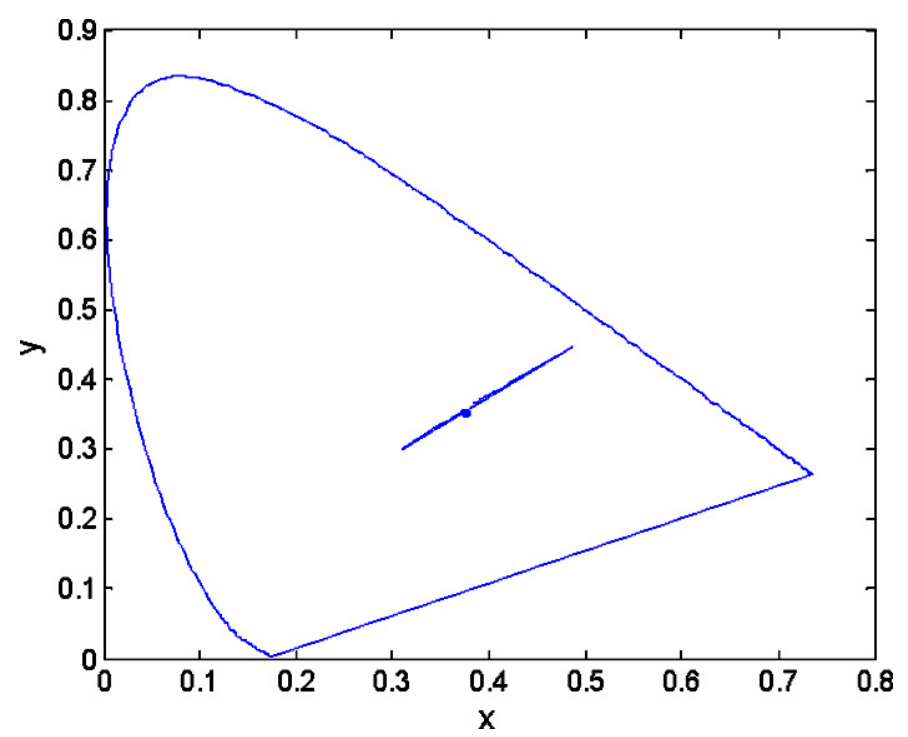

FIGURE 12 - CIE 1931 chromaticity diagram of an IPS-LCD cell using CBC-3 film as the inner protective polarizer films at $40^{\circ}$ of white-light incidence angle (z-CBC Film: $R_{0}=0.2 \mathrm{~nm}, R_{\text {th }}=0.1 \mathrm{~nm}$ ). 


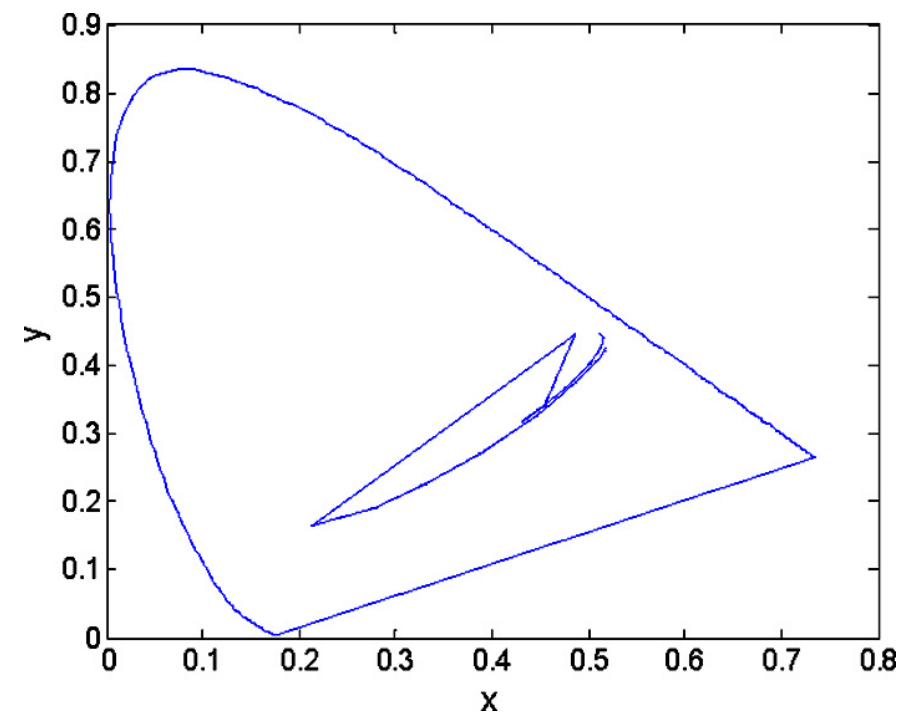

FIGURE 13 - CIE 1931 chromaticity diagram of an IPS-LCD cell using a conventional TAC film as the inner protective polarizer films at $40^{\circ}$ of white-light incidence angle (TAC Film: $R_{0}=5 \mathrm{~nm}, R_{\text {th }}=50 \mathrm{~nm}$ ).

having $R_{0}=5 \mathrm{~nm}$ and $R_{t h}=50 \mathrm{~nm}$ were shown in Fig. 13 . Not surprisingly, a substantially larger degree of color shift occurred at large viewing angles than the case of the CBC-3 film. The average color shift $\left(\Delta u^{\prime} v^{\prime}\right)$ was calculated to be about 0.305 . This is more than twice of the value observed when predicting the performance of the low-retardation CBC-3 film.

In addition to substantially reducing the color shift when using CBC-3 film as the inner protective film of the polarizer, minor improvements on the viewing angle and contrast ratio was also obtained from the optics calculations. Thus, a completely isotropic film such as CBC-3 should provide some real advantages for improving the overall viewing characteristics of an LCD, particularly with respect to IPSLCD design.

\section{Conclusion}

Cyclic block copolymers possess an interesting set of birefringence properties. Both molecular orientation and morphological effects can contribute to the total birefringence. However, due to a close refractive-index matching between the hydrogenated styrene phase and the hydrogenated isoprene phase, the form birefringence due to block-copolymer morphology is found to be only a small fraction of the total birefringence, whereas molecular orientation remains to be the dominant mechanism for the observed birefringence in CBC films, particularly when the polymer is oriented.

By optimizing the comonomer composition, an isotropic film was recently developed using cyclic-block-copolymer technology. This new optical film shows near-zero optical retardation in both the film plane and thickness direction. No measurable wavelength dependence for the retardation property of CBC film was observed. In addition, the CBC film has excellent thermal, mechanical, and barrier properties. Commercial-scale production of CBC film by a melt-extrusion process has also been demonstrated. The use of melt processes for making a polarizer protective film provides a more cost effective and environmental friendly technology than current commercial TAC film manufacturing processes based on solvent casting.

\section{Acknowledgments}

We would like to thank Dr. Ruibo Lu at the University of Central Florida (currently at Pixel Qi) for conducting the optical simulation of CBC film and TAC film. Assistance on the optical measurement from Dr. Yi Jin and Vicki Tharp at The Dow Chemical Company is also acknowledged.

\section{References}

1 F. S. Bates et al., "PCHE-based pentablock copolymers: Evolution of a new plastic," AIChE J. 47, No. 4, 762-765 (2001).

2 M. Yamazaki, "Industrialization and application development of cycloolefin polymer," J. Molecular Catalysis A: Chemical 213, 81-87 (2004).

3 G. Khanarian, "Optical properties of cyclic olefin copolymers," Opt. Eng. 40, 1024 (2001)

4 J. Ruokolainen et al., "Effect of thermal history and microdomain orientation on deformation and fracture properties of poly(cyclohexylethylene)-polyethylene triblock copolymers containing cylindrical PE domains," Macromolecules 35, 9391 (2002).

5 C. Y. Ryu et al., "Chain architecture effects on deformation and fracture of block copolymers with unentangled matrices," Macromolecules 35, 2157 (2002)

6 H. Nakayama et al., "Development of low-retardation TAC film for protective films of LCD's polarizer," J. Photopolym. Sci. Tech. 19, 169 (2006)

7 A. Tagaya et al., "Compensation of the birefringence of a polymer by a birefringent crystal," Science 301, 812 (2003).

8 R. Hahn and J. H. Wendorff, "Compensation method for zero birefringence in oriented polymers," Polymer 26, 1619 (1985).

9 S. Iwata et al., "Transparent zero-birefringence copolymer and its optical properties," Appl. Opt. 36, No. 19, 4549 (1997).

10 D. A. Hucul and S. F. Hahn, "Process for hydrogenating aromatic polymers," U.S. Patent 5,612,422 (1997).

11 D. A. Hucul and S. F. Hahn, "Process for hydrogenating aromatic polymers," U.S. Patent, 5,654,253 (1997).

12 D. A. Hucul and S. F. Hahn, "Process for hydrogenating aromatic polymers," U.S. Patent, 5,700878 (1997).

13 Y. Konishi et al., "Key products development based on cyclo olefin polymer for LCD-TV," Proc. SPIE 6289, 628905 (2006).

$14 \mathrm{~J}$. Li and S-T. Wu, "Two-coefficient Cauchy model for low birefringence liquid crystals," J. Appl. Phys. 96, 170 (2004).

15 W. Kuhn and F. Grun, Kolloid-Z 101, 248 (1942).

16 M. H. Liberman et al., "Stress-optical behavior of polymethylene and poly (dimethylsiloxane)," Macromolecules 5, 550 (1972).

17 I. M. Ward, Structure and Properties of Oriented Polymers, 2nd edn. (Chapman \& Hall, 1997), Chap. 2 and references therein.

18 M. J. Folkes and A. Keller, "The birefringence and mechanical properties of a 'single crystal' from a three-block copolymer," Polymer 12 , 222 (1971).

19 I. Hamley, The Physics of Block Copolymers (Oxford University Press, 1998), Chap. 2 and references therein

20 R. Lu et al., "Ultrawide-View liquid crystal displays," J. Display Technol. 1, 3 (2005). 


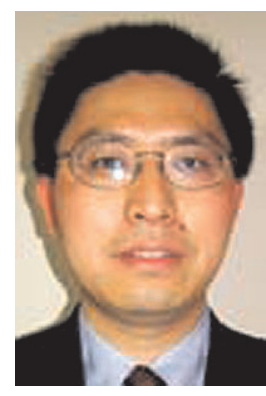

Weijun Zhou received his Ph.D. degree in chemical engineering from California Institute of Technology and his B.S. in materials science and engineering from University of Science and Technology of China. He is currently a Sr. Research Specialist at Core R\&D - New Products Group of The Dow Chemical Company. His current areas of research interest are optical polymer, optical film, structure-properties relationship, and multifunctional materials for electronics and display applications.

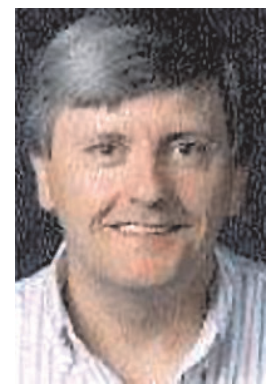

Daniel J. Murray is a Senior Research Specialist in Dow's Core R\&D where he is involved in the synthesis and development of new polymeric systems for a variety of applications. He received his B.S. degree in chemistry from Saginaw Valley State University.

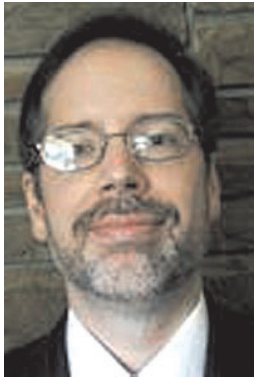

Kurt A. Koppi received his B.S. degree in chemical engineering from the University of Illinois in 1988 and his Ph.D. degree in chemical engineering from the University of Minnesota in 1993. He is currently a Sr. Research Specialist in the New Products Core R\&D Department at The Dow Chemical Company in Midland, MI. His areas of expertise are polymer processing, rheology, and structure-property relationships.

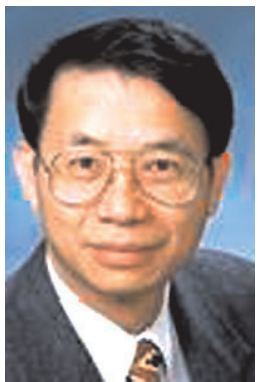

Shin-Tson $\mathbf{W u}$ is a PREP professor at the College of Optics and Photonics, University of Central Florida. Prior to joining UCF in 2001, Dr. Wu worked at Hughes Research Laboratories (Malibu, California) for 18 years. He received his Ph.D. degree from the University of Southern California and his B.S. degree in physics from National Taiwan University. His studies at UCF concentrate in foveated imaging, bio-photonics, optical communications, liquid-crystal displays, and liquid-crystal materials. Dr. Wu is a Fellow of the IEEE, SID, and OSA. He has co-authored 2 books: Reflective Liquid Crystal Displays (Wiley, 2001) and Optics and Nonlinear Optics of Liquid Crystals (World Scientific, 1993). 